\title{
Experimental application of environmental sensitivity in interactive fashion and textile design
}

\begin{abstract}
This paper presents an experimental application of environmental sensitive textiles developed from an ongoing design research project titled The Creation of Interactive Textiles with Adoption of Environmental Sensitivity. The research aims to create value added interactive fashion and textile designs by integrating design creativity, concepts of interactivity, theories of human perception and traditional textile craftsmanship, and further unfolds a discussion on this newly emerged design research area. The experimental attempt, featuring thermo- and photo-sensitivities, was made with the perspectives of critical design practice summarized by Malpass. ${ }^{1}$ Equipped with the reactive properties; the attire is capable to response to the corresponding external stimuli and function as an interactive platform. With reference to the work created, a more in-depth investigation is proposed as the paper conclusion.
\end{abstract}

Volume 2 Issue 4 - 2017

\author{
Haze $\mathrm{Ng}$, Gloria Wong, Kinor Jiang \\ Institute of Textiles and Clothing, The Hong Kong Polytechnic \\ University, Hong Kong
}

\begin{abstract}
Correspondence: Haze $\mathrm{Ng}$, Institute of Textiles and Clothing The Hong Kong Polytechnic University, Room QT715, Q Core, 7/F, Hunghom, Kowloon, Hong Kong, Tel 8529784 I556, Email haze.khng@gmail.com
\end{abstract}

Received: February 14, 2017 | Published: August 03, 2017

Keywords: environmental sensitivity, environmental sensitive textiles, interactive textiles, fashion, textile design

\section{Introduction}

Through introducing ideologies, materials and systems of other disciplines to fashion and textile design, new generation researchers and designers have been exploring the feasibility of contemporary fashion and textiles. Several pioneering experts have provided a number of visionary assemblies of contemporary fashion and textile designs Kettley ${ }^{2}$ Pailes-Friedman ${ }^{3}$ Quinn. ${ }^{4-6}$ It is perceptible from the assemblies that, being investigated proactively, interactive fashion and textile design is one of the prominent hybrid disciplines. However, the development of this newfangled subcategory is notably technologydriven and tilting. Advancement and investigation on other significant design attributes and possibilities are left elementary.

Firstly, it is observable that majority of the pioneering projects are realized by building interactive electronic attachments on fashion and textile creations. The interactive behavior significantly relies on the external, usually computerised, outboard system. The attire and textile substrate are mildly functioning as a trifling part or mantling shell. On the other hand, there are some projects introduced responsive properties to textile substrata and successfully created interactive designs without assistance of external appliance. Applying responsive smart material on textile substrate is one of the key measures. For examples, Woven Light series by Kathy Schicker, Constellation Wallpaper by Aurélie Mossé, Swamp Stools by Erin Hayne and Nuno Goncalves, and Motion Response Sportswear by Kerri Wallace Quinn. ${ }^{6}$ By injecting smart material into textile process, the substrate itself is capable to detect and respond to external stimulus and affords to perform the interactive behavior. Most of the realized projects from this approach cultivate only on a single reactive property. The investigation on multi-reactive substratum and the corresponding design application has not been significantly conducted.

Secondly, for some of the early creations, the level of interactivity is primitive. The perceivable reactions, though brilliant and stimulating, are preset with a fixed simplex result, the user and audience therefore process very limited control and autonomous capability throughout the interaction. Since the reaction possibility afforded is materially defaulted, the human-artefact interactive behavior generally stops at the sensory and merely semantic level Sohn. ${ }^{7}$ The engagement between the designer, the designed and the user is therefore primary and at a certain level alienated. As a result, the expressional and communicative values often derived through bidirectional humanartefact interaction and the corresponding communication context Kiousis, ${ }^{8}$ of the current interactive fashion and textile designs are comparatively low.

Thirdly, while interactive fashion and textiles are being explored intensively with advanced technologies, traditional crafting techniques are very much left neglected. Apart from the promising digitised technologies, some time-honoured craftsmanship, for instances Shibori, K'o-ssu, Ikat and hand embroidery, are also capable to create highly sophisticated and artistic visual tableau for attire and textile design purpose. Some of the unique effects, distinctive textures and adventuristic results are unachievable by the high-tech machinery based technologies. The advantages of the traditional techniques, for instances high level of flexibility and uniqueness, should not be overlooked. However, no significant exploration has been done to integrate the ideology of interactivity with this traditional craftsmanship of skilfulness.

In order to fill the research niche unfolded, the aforementioned research study targets to combine different selected environmental sensitivities to create skillfully crafted hybrid textiles which are capable to perform multiple responsive behaviors, and hence function as an interactive platform. At the early explorative stage of the research, three parallel design development threads with foci located on the investigation of compatible reactive medium, the development of interactive yarns and substrata, and the exploration of applicable textile craftsmanship have been conducted in a divergent manner. ${ }^{9}$ This paper showcases one of the initial cross-thread achievements attained through a critical design practice. The experimental attire titled Textural Dynamics is a hybrid creation reactive to ambient light and temperature. The work was presented in three international exhibitions located in Hong Kong, Oaxaca, Paris and Beijing, and received positive evaluations with an award granted in the 10th International Shibori Symposium. 


\section{Critical design-textural dynamics}

In the early development stage, the initial feasibility trials on hybrid sensitivity integration at different sequential textile processes, including pre-, mid- and post-fabric-formation, have been conducted and positively proven. ${ }^{10}$ In order to put the trial results in actual practice, a prototype was artfully created with enhanced design sophistication. Aiming to suggest a new direction of consideration in the interactive fashion and textile design discipline and unfold a platform for discussion, the prototype is designed with the theoretical perspectives of the newly sprang critical design practice narrated by Mazé \& Redström; ${ }^{11}$ Malpass.

Deliberating the propositions of scholars and researchers from different design disciplines, Malpass ${ }^{1}$ has introduced concordantly three theoretical perspectives for critical design practice as Parafunctionality, Post-optimal design and the Aesthetics of Use. Instead of focusing on the orthodox functionality of a design, the practice takes an exploratory and proactive leap to project a redolent space inducing diverse supreme values through discussions and debates. The practice attempts to explore possibilities and potential alternatives to attain a poetic experience through interaction, both actual and rhetorical, between the design and the user. The poetic proposition stimulates the user's imaginative faculty and invites the user to actively co-construct an aesthetic experience with a series of subjective interpretations and reactions. It further explores, with existentialist perspective, into the meaning and relationship constructed between the design and the user in the actual encounter and engagement.

\section{Objectives}

Sharing a similar philosophical stance, the constructive design research takes a critical and speculative perspective in interactive fashion and textile design. The research prototype Textural Dynamics aims to function as Mazé \& Redström ${ }^{11}$ suggestion of diversifying the thinking patterns and dimensions of the researchers and designers in understanding the design problems, ideas and boundaries. In preference to imposing a concrete and certain perceivable reaction, the design embraces engagement uncertainty and potential ambiguity Malpass ${ }^{1}$ by providing the user and audience an interactive platform with a higher level of autonomy and flexibility. The research prototype attempts to inject the aesthetic attribute into the process of design completion. Facilitated with appropriate tools, the design invites the wearer and audience to perform actively the role of coauthor. The attire reacts dynamically to external stimuli, including ambient temperature and light, which are adjustable by the wearer and audience, and evolves into different appearances. With the involvement of different parties, the attire is poetically completed.

The specific objectives of the research prototype Textural Dynamics are as follows:

a. To apply dual environmental sensitivity in interactive fashion and textile design

b. To integrate the ideology of interactivity with traditional Shibori technique

c. To construct an interactive platform with a considerably higher level of user autonomy

d. To diversify the consideration perspectives in understanding interactive fashion and textile design problems, ideas and boundaries

e. To unfold discussion and debate on the disciplinary practice and corresponding methodology of interactive fashion and textile

\section{design Mazé \& Redström ${ }^{11}$}

f. To serve as a supportive research prototype, subject to evaluation, for the overall constructive design research.

\section{Design practice}

The actual design practice of this experimental application involves a series of design methods including literature review, brainstorming, sketching, preliminary prototyping, and design documenting and research prototyping. The design methods were conducted with an universal design process delimited by Koberg \& Bagnall, ${ }^{12}$ which consists of the seven steps namely, situation acceptance, analysis, definition, ideation, selection, implementation and evaluation. Initially, the design processes were established step by step, however, as the practice developed, the proceeding was not limited nor forced according to the achievements and failures of each stage. In preference to a linear sequence, the model was adopted holistically as an inspirational action guiding protocol with the stages developing concurrently and interrelated under a repeating manner in order to attain situational optimal conditions.

Since the design practice is part of the parent research, prior to this phase, a series of literature reviews has already been conducted in five major scopes to assemble existing knowledge and understanding on

i. The ideology of interactivity and the related philosophies and theories

\section{ii. Design research methodologies}

iii. Realized pioneering interactive textile design projects

iv. Technical properties of the environmental sensitive materials and

v. Potential traditional textile craftsmanship.

The focus of the review specifically done for this phase is located on the traditional craftsmanship of Shibori. Functioning as an initiator, the literature review helps the researcher to construct fundamental knowledge on the acquired techniques, which further induces the formulation of the other design methods. Through an intensive knowledge assimilation and self-position identification, the researcher then engaged the technique with depth by brainstorming, sketching, preliminary prototyping and design documenting.

Brainstorming was conducted to perform as a semi-systematic possibility seeking tool. Potential discovering and creativity generating are the major foci aiming to maximize the multidimensional imagination on the design possibility. It also functioned to generate and accumulate positive emotion and fascination on the research area. Two divergent threads were initiated in exploring material possibilities and potential Shibori techniques in forms of mindmapping and rapid ideation (Figure 1). The process was associated with intensive rough sketching and simple hands-on mock-up. Three mind-mapping techniques were adopted in accordance to different natures of idea generation. The subject matters, for instances different types of Shibori and smart materials, were firstly expanded by lateral relating. Different themes, for examples significant property, independent variable, possible production stage and available resources, were assigned and linked to the same core. Secondly, the prominent products of the lateral relating were expanded with question words and related considerations for rapid ideation. With a number of thematically formed and question-led mind-maps, secondary and tertiary mind-maps were then drawn by intersecting the idea networks constructed from the previous generations, followed by detailed analyses and initial proposals (Figure 2). 
Staged at the early part of the design practice, sketching and preliminary prototyping aim to perform as visual and physical explorations engaging the researcher with the possibilities unfolded from the previous literature review and brainstorming phases. With the objective to embrace experimental uncertainty and unexpected windfall, there is no specific limitation and restriction preset. In preference to working cautiously with restrictive design specifications and justifications, the researcher set free to absorb firsthand experience on the available materials and potential craftsmanship, and further familiarized with the skills and processes through intensive practices. A series of mono-sensitive swatches (Figure 3) were achieved and documented with imagery records with detailed descriptions on the material contents, practice measures and results achieved. The results were analysed, with the researcher's subjective interpretation, in respect to the capability of affording the perceived, technical and contextual interactivity Kiousis ${ }^{8}$ deliberated in the earlier research stage.

By intersecting the independent experimental trials with each other, the prototyping phase was then proceeded to the secondary level resulting in a dual-sensitive swatch. ${ }^{13}$ Step by step, the transparent polyester organza was coated with azure thermo chromic pigments and transparent-green luminescent coating on the right and wrong side respectively. The fabric was then gathered and secured by rolls of running stitches similar to the resisting mechanism of the traditional Japanese textile craftsmanship, Nui Shibori. Another layer of transparent-azure luminescent coating with higher viscosity was applied onto the surface of the gathered panel. Cracks, which allow the transparent-green luminescent coating on the wrong side of the fabric to be visible from the right side, were created on the azure thermo chromic layer along the gathers as a result of imbalanced viscosity between coatings. The hybrid swatch developed reacts to ambient thermo- and photo- stimuli and displays dynamically different visuals. The surface changes from shades of azure to light grey in contact with heat, and reacts to ultraviolet light resulting in temporary glowing surface of luminescent azure and green. Proven feasible by the secondary prototyping, the procedures and mechanism developed were adopted in the research prototyping phase.

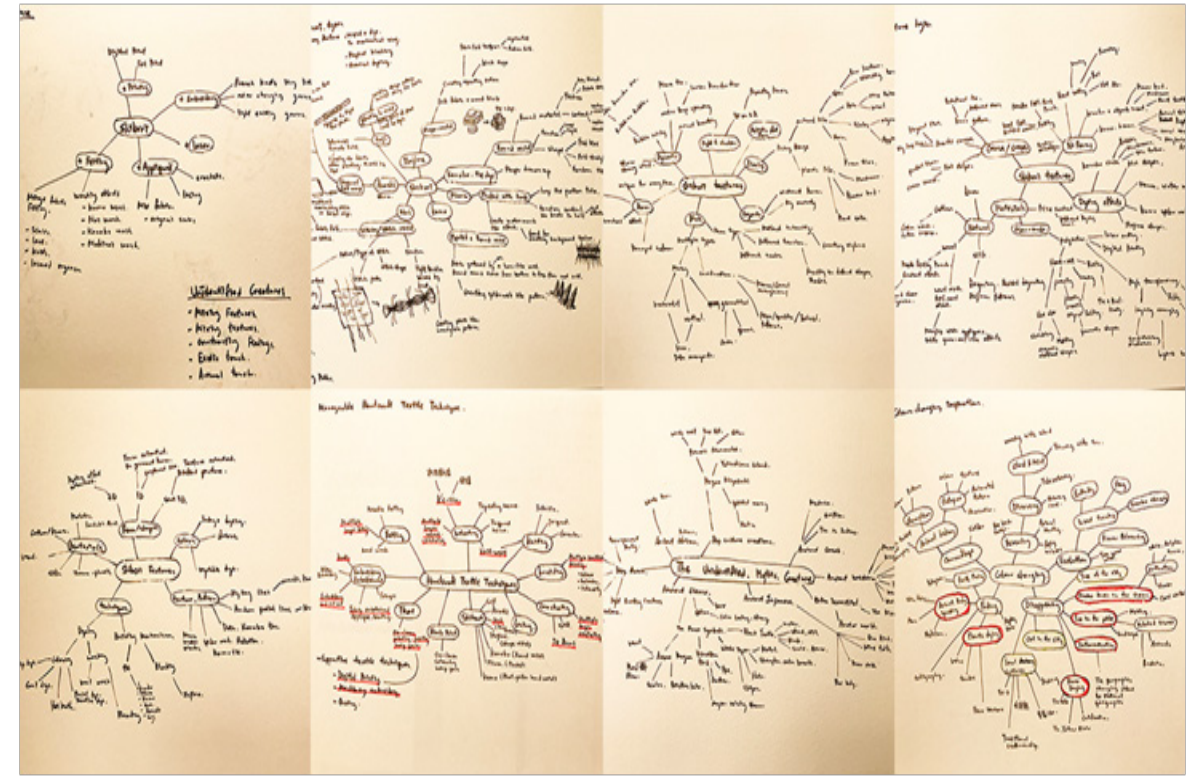

Figure I Mind-mapping and rapid ideation.

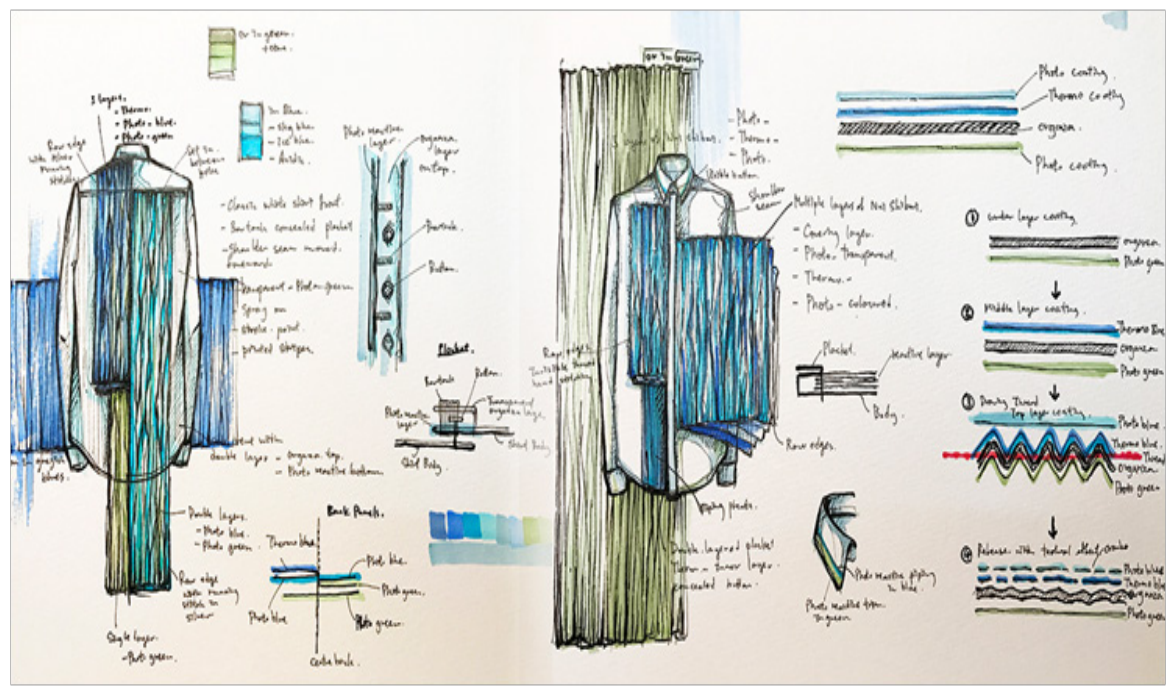

Figure 2 Examples of initial proposals. 


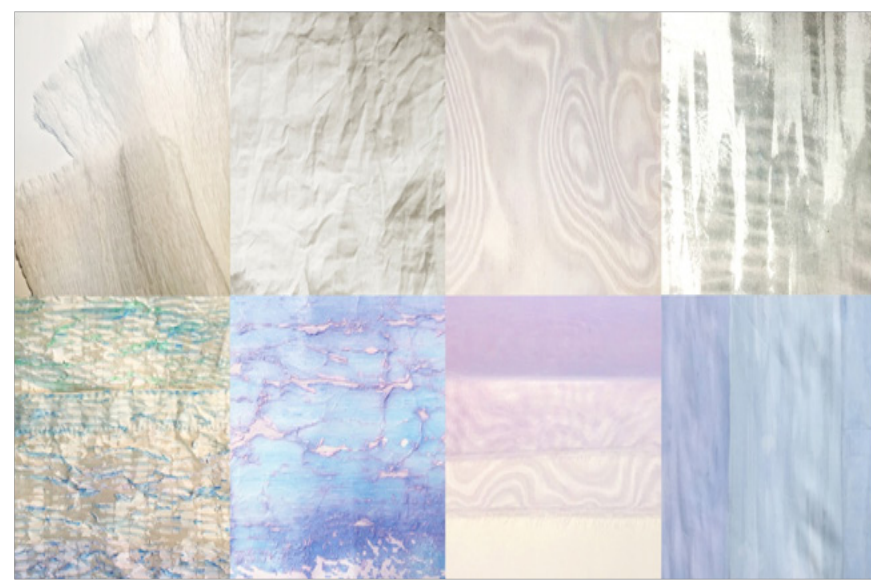

Figure 3 Mono-sensitive swatches developed.

After considering the situational suitability, a selected initial proposal was refined by integrating the results attained in the multisensitive textile development process. Supported by the previous stages, the research prototyping phase was smoothly conducted with enhanced design sophistication. A dual-sensitive panel (Figure 4) was recreated with additional hand embroidery and texture enhanced layer and further applied to the critical design practice resulting in the research prototype, Textural Dynamics (Figure 5). Functioning as an in-stage implementation, the attire was cautiously designed and artfully created to give a physical form to the established idea in a balanced, pragmatic and holistic practice. Figure 6 shows the different reactive behaviors and hence the diverse appearances that the realized prototype affords. Equipped with the right exhibition setting and tools, for example, ultraviolet laser pointer, the audience is entitled a high level of user autonomy. Since the audience is able to manipulate the appearance of the attire and temporarily compose a customized signifier, a higher level of interactivity, thus, an enhanced human-artefact engagement and the corresponding user-experience are anticipated. Nevertheless, the hypothesis is to be proven by further empirical study.

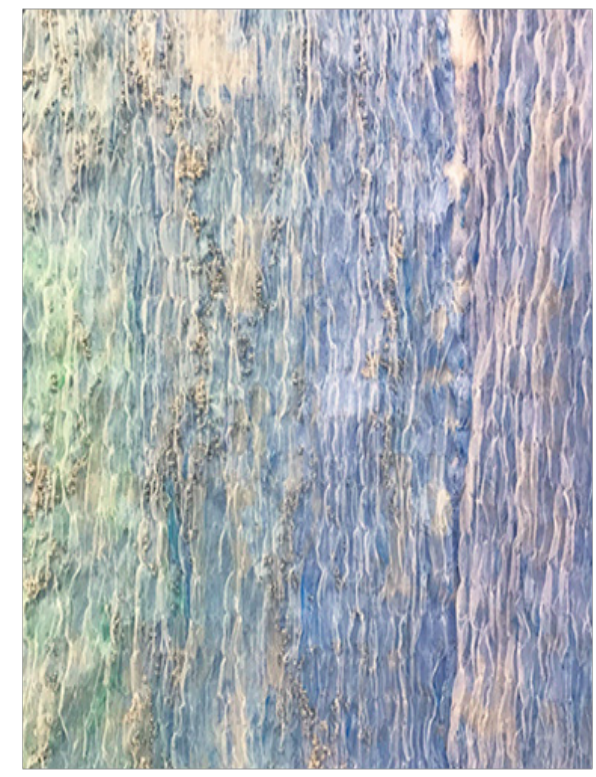

Figure 4 Dual-sensitive panel.

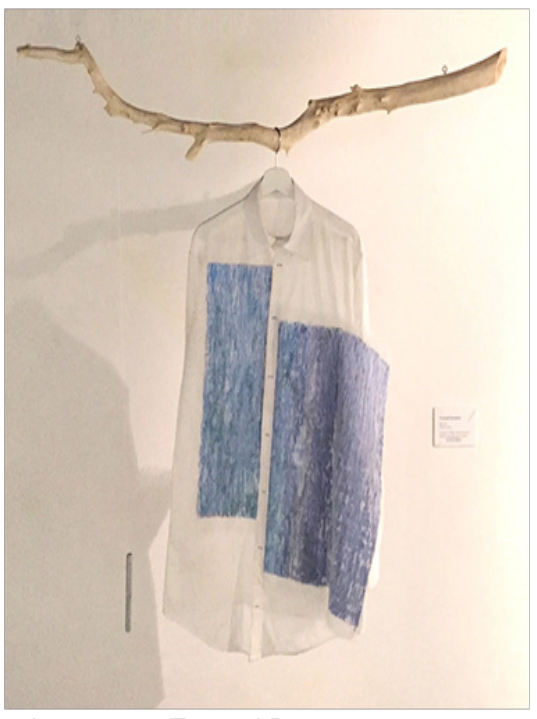

Figure 5 Research prototype Textural Dynamics.

\section{Conclusion}

In addition to the mono-responsive creations realized by other pioneering researchers, this experimental application has further created the hybrid attire which is capable to present multiple appearances in contact with different environmental and artificial stimuli. This paper shows the feasibility of combining photo- and thermo-sensitivity with an idea integration of a time-honoured traditional textile craftsmanship. The attempts made here are subject to further improvement. More advanced investigations are being conducted to explore the relationship between different parameters, for instances the viscosity, concentration and thickness of the reactive media, the construction of the crafted textile substrata and the corresponding interactive performances.

In addition to the prototype presented in this paper, several parallel experimental threads have been unfolded to investigate other possible environmental sensitive materials for the on-going parent research, for example hydro-reactive textiles. Consolidating the achievements of each thread, it is highly feasible to introduce more types of environmental sensitivity to interactive fashion and textile design practices, realizing triple or even more sensitive creations. In other words, a single textile surface will be able to afford and present multiple and flexible signifiers, and hence poetically lead to different signified through series of signification

Barthes, ${ }^{14}$ Barnard ${ }^{15}$ enriching human-artefact interactions. In order to proceed to the evaluation stage, a collection of interactive fashion and textile designs are being actualized.

In order to critique and examine the realized creations, an evaluation stage is briefly proposed here. Since the significant outcomes of the constructive design research are physical creations, usually suitable and decent for exhibition, a showroom based evaluation approach is believed to be appropriate. Observed from the exhibition in the Fashion Gallery of the Hong Kong Polytechnic University, and the imagery records of the exhibitions in Oaxaca and Paris, audiences were found positively attracted by the dual interactive properties of the prototype (Figure 7).

As per Kiousis ${ }^{8}$ advised, multiple measures should be applied for 
evaluating the total degree of interactivity, since the fundamental natures of the attributes are different. By formulating different measures, a triangulated showroom based case study, including direct observation, questionnaire, semi-structured interview and expert interview, is suggested. The case study aims to collect dominating qualitative and subordinate quantitative data, followed by content and statistic analyses respectively.

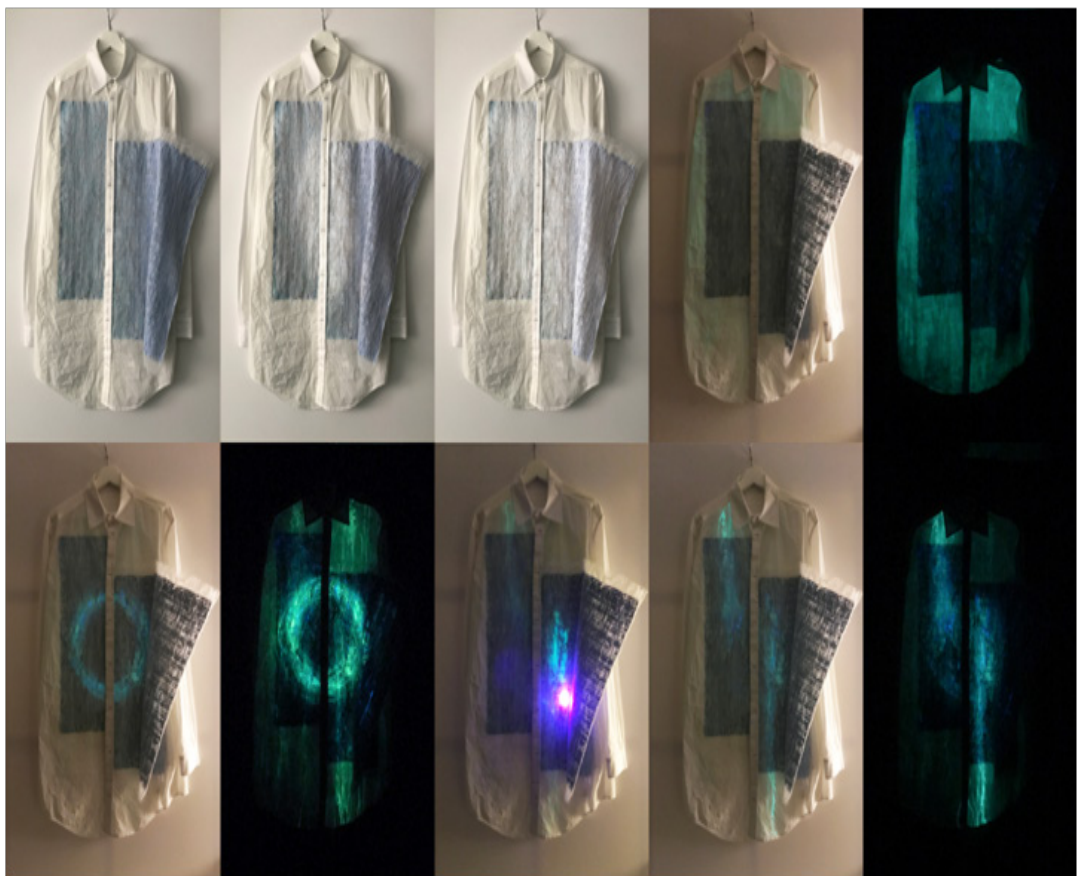

Figure 6 Textural Dynamics under different environmental conditions.

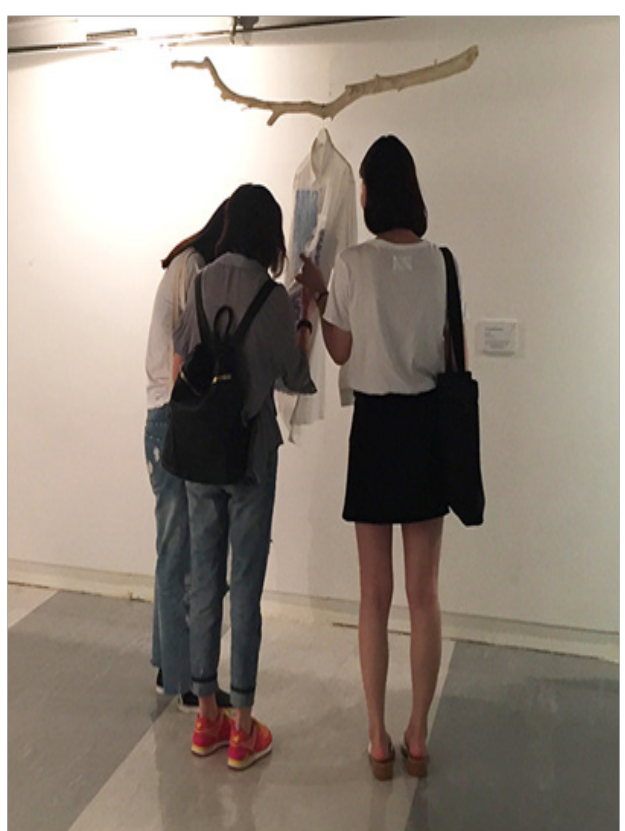

Figure 7 Audiences actively interacting with Textural Dynamics in exhibition.

The direct observation is proposed to be done simultaneously with the semi-structured interview and expert interview. The human-artefact interaction will be observed with a preset observation protocol when the subjects are presented with the realized creations. The observation foci are located on the person, objects, situation, time and activity. A questionnaire will be given to the exhibition audiences in order to collect numerical ratings of the interactivity perceived. Subjects selected with convenience sampling will be invited to participate in the semi-structured interview. The interview targets to gather indepth qualitative data on human perception, interpretation, interaction and criticism on the designs. Furthermore, expert interviews will be formulated with the objective to generate competent and critical opinion from the related professionals. Structured with simple guidance on the critique dimensions, the interviewees will be given a high level of flexibility and large room for professional judgements. The data gathered through the proposed approach are expected to function as complementary causes for triangulation or initiation confirming or rejecting the research hypotheses.

With the ambiguity-embracing theoretical stance Malpass ${ }^{1}$ of critical design practice, the findings in the evaluation stage, regardless to the dispositions in favour of or rejecting the research hypotheses, will be contributive to the discussions and debates on the subject matter. The insights and propositions generated from the evaluation and discourses will provide design navigations for future development of the interactive fashion and textile design discipline.

\section{Acknowledgements}

The authors sincerely acknowledge the support from Institute of Textiles \& Clothing, The Hong Kong Polytechnic University.

\section{Conflict of interest}

The work presented in this paper is part of the research study titled The Creation of Interactive Textiles with Adoption of Environmental Sensitivity, which is funded by The Hong Kong Polytechnic University. No other conflict of interest exists. 


\section{References}

1. Malpass M. Critical design practice: Theoretical perspectives and methods of engagement. The Design J. 2016;19(3):473-489.

2. Kettley S. Designing with Smart Textiles. 1st ed. London: Bloomsbury Publishing Plc; 2016. p. 240.

3. Pailnes-Friedman R. Smart Textiles for Designers. London: Laurence King Publishing Ltd; 2016. p. 192.

4. Quinn B. Textile Futures. 1st ed. New York: Berg Publishers; 2010.

5. Quinn B. Fashion Future. London: Merrell Publishers Ltd; 2012. p. 240.

6. Quinn B. Textile Visionaries.London: Laurence King Publishing Ltd; 2013. p. 312.

7. Sohn D. Anatomy of interaction experience: Distinguishing sensory, semantic, and behavioural dimension of interactivity. New Media \& Society. 2004;13(8):1320-1335.

8. Kiousis S. Interactivity: A concept explication. New Media \& Society. 2002;4(3):355-383.

9. Ng H, Wong G, Jiang K. Development of Interactive Textiles with Dual Environmental Sensitivity. The 14th Asian Textile Conference, Hong Kong; 2017.
10. Ng H, Wong G, Jiang K. The Development of Interactive Textiles with Multiple Environmental Sensitivities. The 9th Textile Bioengineering and Informatics Symposium-The 6th Asian Protective Clothing Conference. Melbourne, Australia; 2016.

11. Mazé, R, Redström J. Difficult forms: Critical practices in design and research. Conference of the International Association of Societies of Design Research. Hong Kong, 2007. p. 1-18.

12. Koberg D, Bagnall J. The Universal Traveler: A Soft-systems Guide to Creativity, Problem-solving and the Process of Reaching Goals. USA: Crisp Publications Inc; 1974. p. 148.

13. Ng H, Wong G, Jiang K. Experimental Application of Dual Environmental Sensitivity on Interactive Textile Design. The $7^{\text {th }}$ International Conference of Applied Research on Textiles, Hammamet, Tunisia; 2016.

14. Barthes R. The Fashion System. London: Jonathan Cape Ltd; 1964. p. $1-233$.

15. Barnard M. Fashion as Communication. London: Routledge; 2002. p. 1-7. 